\title{
Latanoprost and Dorzolamide for the Treatment of Pediatric Glaucoma: The Glaucoma Italian Pediatric Study (Gipsy), Design and Baseline Characteristics
}

\author{
Luciano Quaranta · Elena Biagioli · Francesca Galli · Davide Poli • \\ Eliana Rulli · Ivano Riva · Lital Hollander • Andreas Katsanos • \\ Antonio Longo · Maurizio G. Uva · Valter Torri · Robert N. Weinreb
}

Received: May 4, 2016/ Published online: June 16, 2016

(C) The Author(s) 2016. This article is published with open access at Springerlink.com

\section{ABSTRACT}

Introduction: To investigate the efficacy of a treatment strategy with latanoprost and dorzolamide in primary pediatric glaucoma patients partially responsive to surgery.

Enhanced content To view enhanced content for this article go to http://www.medengine.com/Redeem/98D4 F06077358131.

L. Quaranta · I. Riva

Department of Medical and Surgical Specialties Section of Ophthalmology, University of Brescia, Brescia, Italy

E. Biagioli $(\varangle) \cdot$ F. Galli $\cdot$ D. Poli · E. Rulli ·

L. Hollander · V. Torri

Laboratory of Methodology for Clinical Research, IRCCS - Istituto di Ricerche Farmacologiche Mario Negri, Milan, Italy

e-mail: elena.biagioli@marionegri.it

A. Katsanos

Department of Ophthalmology, University of Ioannina, Ioannina, Greece

A. Longo - M. G. Uva

Azienda Ospedaliera Universitaria, "Policlinico Vittorio Emanuele", P.O. Gaspare Rodolico, Catania, Italy

R. N. Weinreb

Department of Ophthalmology, Shiley Eye Institute, Hamilton Glaucoma Center, University of California San Diego, San Diego, CA, USA
Methods: Single arm, prospective, interventional multicenter study. Primary pediatric glaucoma patients younger than 13 years after a single surgical procedure with IOP between 22 and $26 \mathrm{mmHg}$ were considered eligible. At baseline, patients were allocated to latanoprost monotherapy once daily. Depending on intraocular pressure (IOP) reduction at first visit, the patients were allocated to one of three groups: continuation of latanoprost monotherapy, addition of dorzolamide twice daily, or switch to dorzolamide three times daily. The same approach for allocation in medication groups was used in all subsequent visits. Patients in the dorzolamide monotherapy group with IOP reduction $<20 \%$ from baseline were considered non-responders and withdrawn. Study treatment and patient follow-up will continue for 3 years or until treatment failure. The primary endpoint is the percentage of responders. Secondary endpoints are time to treatment failure and frequency of adverse events.

Results: A total of 37 patients (69 eyes) were enrolled. The mean age was $4.0 \pm 3.8$ years, the female/male ratio was $1 / 1.7$, and the majority of 
patients were Caucasian. Eighty percent of patients had bilateral glaucoma. Goniotomy was the most frequently performed surgery (38.6\%), followed by trabeculotomy (22.8\%), trabeculectomy $(21.1 \%)$, and trabeculectomy plus trabeculotomy (17.5\%). The baseline IOP was $23.6 \pm 1.5 \mathrm{mmHg}$.

Conclusion: The study population is representative of patients frequently encountered after the first surgery for primary pediatric glaucoma. The study will produce evidence on the medium-term efficacy of a defined pharmacological approach.

Keywords: Dorzolamide; Latanoprost; Medical therapy; Ophthalmology; Pediatric glaucoma; Surgery

\section{INTRODUCTION}

Pediatric glaucoma represents a group of potentially blinding diseases characterized by elevated intraocular pressure (IOP). The prognosis is largely dependent on early, accurate diagnosis and successful treatment that lowers IOP to levels where progression is unlikely [1]. Due to the rarity of these glaucomas, epidemiologic and prospective randomized studies are difficult to undertake.

According to the latest classification proposed by the World Glaucoma Association Consensus group, pediatric glaucoma is classified as primary or secondary [2]. In this classification, the term primary refers to glaucomas caused by anomaly of filtration angle, while secondary pediatric glaucoma is further classified according to whether the disease is acquired after birth or is present at birth (non-acquired). Non-acquired pediatric glaucoma is categorized according to whether the signs are mainly ocular or systemic.
Although surgery is the definitive treatment of choice for patients with pediatric glaucoma [3-11], topical medications are usually also needed as temporary treatment before surgery or as adjunctive postoperative therapy $[12,13]$. Beta blockers, carbonic anhydrase inhibitors (CAIs) and prostaglandins have all been used in the treatment of pediatric glaucomas [14-16]. Raber and colleagues reported on the safety of latanoprost exposure in a phase 1 study in pediatric and adult glaucoma patients [17]. The systemic exposure to latanoprost acid appeared higher in children than in adults, and suggested that this was due to lower body weight and a small blood volume in children [17]. This higher systemic exposure in children was not accompanied by adverse events, and thus there was an adequate safety margin for the use of an adult topical dose of latanoprost in children [17]. In a 12-week study, with pediatric glaucoma patients, latanoprost and $0.5 \%$ timolol were found to have similar efficacy and tolerability. Mean IOP reductions for latanoprost and timolol after 12 weeks were 7.2 and $5.7 \mathrm{mmHg}$, respectively. However, responder rates (subjects with IOP reduction of at least $15 \%$ ) appeared to be low (60\% for latanoprost and 52\% for timolol) [18].

Latanoprost and dorzolamide are commonly used in pediatric glaucoma patients due to their favorable efficacy and safety profile [14-17, 19-22]. At present, no data are available from prospective clinical trials on the efficacy and safety of a defined therapeutic strategy for the treatment of primary pediatric glaucoma (PPG). The Glaucoma Italian Pediatric Study (GIPSy) investigated the use of a specific therapeutic algorithm with latanoprost and dorzolamide in pediatric glaucoma patients partially responsive to surgery. This article reports the study protocol and baseline characteristics of study participants. 


\section{METHODS}

\section{Study Objective and Design}

The GIPSy is a single arm, prospective, interventional study evaluating the IOP lowering effect of latanoprost and dorzolamide in PPG partially responsive to surgery.

\section{Study Organization}

Patient recruitment was performed in two institutions in Italy: Dipartimento di Scienze Neurologiche e della Visione, USVD "Centro per lo studio del Glaucoma", A.O "Spedali Civili di Brescia" in Brescia and U.O. Oculistica, A.O. Universitaria, "Policlinico Vittorio Emanuele", P.O. Gaspare Rodolico in Catania. The study was conducted in accordance with the Declaration of Helsinki of 1964 as revised in 2013. The study protocol was approved by the Institutional Review Board of each centre. Parents provided written informed consent prior to any study-related activities. The investigation was registered with clinicaltrials.gov (NCT01527682) and was supported by a grant from the Italian Medicines Agency (AIFA - FARM7CZ54W 2007).

\section{Patient Eligibility Criteria}

Eligible patients were younger than 13 years with unilateral or bilateral PPG and IOP levels between 22 and $26 \mathrm{mmHg}$ following a single surgical procedure. PPG was defined by the presence of two or more of the following criteria: IOP $>21 \mathrm{~mm} \mathrm{Hg}$, optic disc cupping (neuroretinal rim narrowing); corneal findings: Haab striae, corneal edema or diameter $\geq 11$ in newborn, $>12 \mathrm{~mm}$ in child $<1$ year of age, $>13 \mathrm{~mm}$ any age; progressive myopia or myopic shift coupled with an increase in ocular dimensions out of keeping with normal growth; and a reproducible visual field defect that is consistent with glaucomatous optic neuropathy with no other observable reason for visual field defect [23].

Children with secondary glaucoma (Sturge-Weber syndrome, type I neurofibromatosis, retinoblastoma, steroid-induced glaucoma, etc.), or with abnormalities of the anterior segment of the eye (cataract, iridocorneal dysgenesis, congenital ectropion uveae, etc.) or children previously treated with the study drugs were not eligible. Children with concomitant systemic diseases (asthma, hypertension, cardiac disease, renal failure, etc.) requiring pharmacological treatment with IOP-modifying drugs (i.e., corticosteroids, beta-blockers, diuretics) or with corneal abnormalities that could influence IOP readings were excluded from the study.

The following operations were allowed in the study: goniotomy, trabeculotomy, trabeculectomy with adjunctive mitomycin C, and trabeculectomy plus trabeculotomy with adjunctive mitomycin C. Study treatment was to be initiated at least 1 month after surgery. Topical steroids were stopped 1-2 weeks post-operatively following goniotomy and trabeculotomy, and continued 4 times a day for 3 months following trabeculectomy and trabeculectomy plus trabeculotomy with adjunctive mitomycin C. All necessary maneuvers (massage, remove or loosen sutures, conjunctival bleb needling) were performed before enrolling patients for the trial.

\section{Procedures}

Eligible patients had a baseline clinical evaluation that included IOP measurement [sitting position: Goldmann applanation tonometer (Haag-Streit, Bern, Switzerland); supine position: Perkins applanation 
tonometer (Clement Clarke MK2, Harlow, England)], central corneal thickness (CCT) measurement with ultrasound pachymeter (DGH 500, Pachette ${ }^{\mathrm{TM}}$; DGH Technology, Exton, USA), anterior segment photography for eyelash growth and iris color, and optic disc photography. Two experienced examiners performed all IOP measurements. Tonometry was performed in the supine position under general anesthesia in non-collaborative children. General anesthesia was obtained by small dose of ketamine and IOP was measured as soon as possible after aesthesia induction [24]. The exact time of IOP measurement was recorded. These examinations were repeated in all subsequent study visits. At the end of the baseline visit patients were prescribed branded latanoprost $0.005 \%$ eye drops (Xalatan $0.005 \%$ eye drops solution; Pfizer Italia) to be instilled once daily at bedtime (2100 hours). The parents of enrolled children were instructed on the appropriate technique of eye-drop administration. The importance of timely instillation of eye-drops followed by eyelid closure and punctual occlusion was stressed.

The first study visit was performed approximately 30 days after commencement of treatment. IOP was measured at the same hour as the baseline visit. If IOP decreased by $\geq 20 \%$ with respect to baseline, the patient was instructed to continue treatment with latanoprost alone. In cases with IOP decrease between 10 and 19\% from baseline, branded dorzolamide $2 \%$ ophthalmic solution (Trusopt $2 \%$ eye drops solution; MSD Italia) twice a day (0800 and 2000 hours) was added to latanoprost (2100 hours). Finally, in cases with IOP decrease $<10 \%$ from baseline or in cases with IOP elevation, treatment with latanoprost was discontinued and dorzolamide $2 \%$ ophthalmic solution was prescribed three times daily (0800, 1400 and 2000 hours).
The second study visit was performed 60 days after the baseline visit. If the IOP decrease was major or equal to $20 \%$ with respect to baseline, treatment was continued. Otherwise, the same approach described in visit 1 was followed. Eyes failing to reach a $20 \%$ IOP decrease from baseline under dorzolamide monotherapy were considered "non-responders", and treated at the physician's discretion. The treatment flow-chart is depicted in Fig. 1.

The following visits were scheduled approximately every 60 days in the first year, every 90 days in the second year and every 120 days in the third year. The same algorithm, described above, for the allocation of patients in drug groups was followed in the following visits. The study treatment continued for 3 years or until treatment failure, whichever occurred first. If the patient was still in treatment at 3 years, a final visit was performed and the study was considered concluded.

\section{Endpoints}

The primary efficacy endpoint, calculated on a per-eye basis, was the percentage of "responders", defined as eyes with IOP reduction of at least $20 \%$ with respect to baseline. Secondary efficacy endpoint was the time to treatment failure, calculated as the time from the date of baseline visit to the date in which the medical treatment was discontinued due to failure to maintain adequate reduction of IOP.

Safety was assessed by evaluating the occurrence of serious and non-serious adverse events in general, and of the following events in particular: excessive eyelash growth (hypertrichosis), alterations of iris color, corneal epitheliopathy, allergic conjunctivitis and CCT changes. 


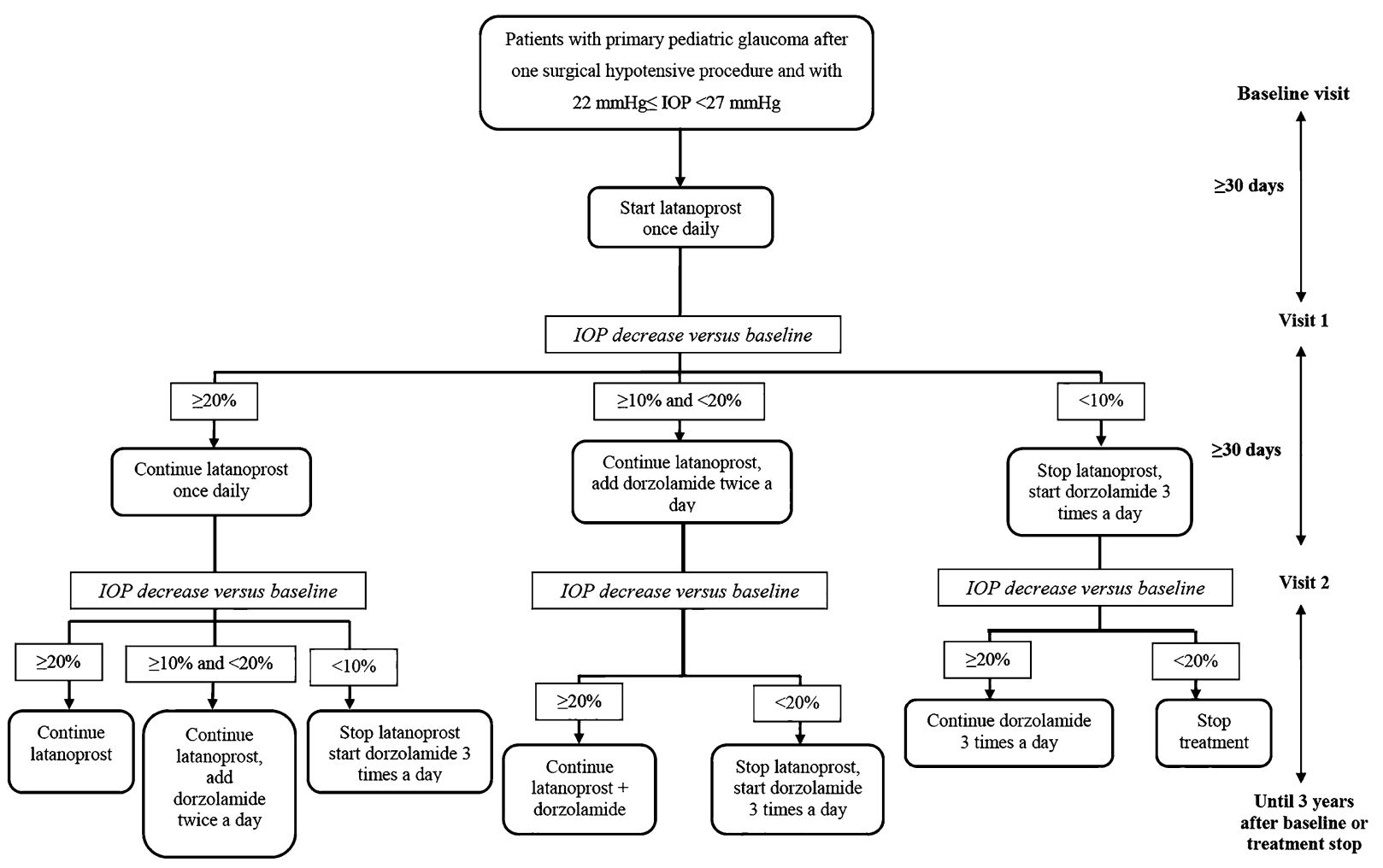

Fig. 1 Flow-chart of study treatment schedule

\section{Statistical Considerations}

\section{Statistical Methods}

The per-protocol population included only eyes without major violation of eligibility criteria that received treatment according to the protocol schedule. All statistical analyses were based on the per-protocol population. Continuous variables were described using mean and standard deviation (SD), while categorical variables were described by means of frequencies and percentages of patients/eyes. The denominator for the percentage calculation was based on the total number of patients/eyes in each relevant analysis group. In all analyses, a $p$ value $<0.05$ was considered significant. Statistical analyses were performed using the SAS $^{\circledR}$ System (v.9.4).

\section{Sample Size Estimation}

The sample size calculation was based on the primary endpoint. Using a Fleming single stage design, according to the A'Hern approach, setting the probability of erroneously concluding that the "responder" rate is greater than $35 \%$ at $5 \%$ (one-sided alpha $=0.05$ ) and the probability of correctly concluding that the "responder" rate is at least $50 \%$ at $80 \%$ (beta error $=0.20$ ), it was found necessary to include 68 eyes. The minimum number of "responder" eyes was set at 31 out of 68 , since this result is associated with a lower limit of the $90 \%$ exact confidence interval (CI) of $35.2 \%$. Since the number of eyes excluded from the per-protocol population was greater than expected, to analyze the primary outcome a recalculation of the achievable statistical power considering the actual number of analyzable eyes is needed. 


\section{RESULTS}

From July 2009 to January 2014, a total of 37 patients (69 eyes) were enrolled: 20 from the Brescia centre and 17 from the Catania centre. Out of 69 eyes, 4 were excluded from the per-protocol analysis because they had not undergone surgery and 8 because they were not treated with the study drugs. Therefore, 57 eyes of 35 patients were analyzed and are described in this report.

Table 1 reports the participants' general demographic characteristics. Mean age was 4 years (SD 3.8), 62.9\% were males, and the majority of patients were Caucasian (91.4\%). Since 28 patients $(80.0 \%)$ had bilateral glaucoma but only 22 patients $(62.9 \%)$ had both eyes eligible, the total number of eyes was 57.

The mean IOP before surgery was $27.9 \mathrm{mmHg}$ (range 20.0-46.0) and the CCT had a mean value of $558 \mu \mathrm{m}$ (SD 47; range 485-665 $\mu \mathrm{m})$. Angle surgery had been

Table 1 Patients demographic characteristics $(n=35)$

\begin{tabular}{ll}
\hline Age (years) & \\
Mean (SD) & $4.0(3.8)$ \\
Min-max & $0.1-13.7$ \\
Sex, $n$ (\%) & $22(62.9)$ \\
Male & $13(37.1)$ \\
Female & $32(91.4)$ \\
Ethnicity, $n$ (\%) & $3(8.6)$ \\
Caucasian & $28(80.0)$ \\
African & $22(62.9)$ \\
Bilateral glaucoma, $n(\%)$ & \\
Both eyes analyzed, $n(\%)$ & \\
\hline$S D$ standard deviation, Min-max minimum-maximum \\
values
\end{tabular}

performed in 35 eyes and filtration surgery in 22 eyes. Goniotomy was the most frequent surgical procedure, performed in 22 eyes (38.6\%), followed by trabeculotomy performed in 12 eyes (22.8\%). Regarding filtration surgery, trabeculectomy and trabeculectomy plus trabeculotomy had been performed in 12 $(21.1 \%)$ and 10 (17.5\%) eyes, respectively (Table 2).

The choice of glaucoma surgical treatment was based on disease severity and age at onset of the disease. Patients with glaucoma at birth and/or cloudy cornea were treated with trabeculectomy alone or combined trabeculectomy and trabeculotomy. Patients

Table 2 Preoperative ocular characteristics $(n=57)$

\section{Preoperative values}

IOP $(\mathrm{mmHg})$

$\begin{array}{ll}\text { Mean }(\mathrm{SD}) & 27.9(3.9) \\ \text { Min-max } & 20.0-46.0\end{array}$

Vertical/horizontal corneal diameter $(\mathrm{mm})$

Mean (SD)

$13.4(1.0) / 13.5$

Min-max

12.0-16.0/

$11.5-16.0$

Central corneal thickness $(\mu \mathrm{m})$

Mean (SD)

$558(47)$

Min-max

485-665

Surgery type, $n$ (\%)

Goniotomy

$22(38.6)$

Trabeculotomy

13 (22.8)

Trabeculectomy

$12(21.1)$

MMC + trabeculotomy

Trabeculectomy MMC

$10(17.5)$

SD standard deviation, Min-max minimum-maximum values 
Table 3 Age and intra-ocular pressure at the time of surgery

\begin{tabular}{|c|c|c|c|c|}
\hline & \multicolumn{2}{|c|}{ Pre-operative IOP $(\mathrm{mmHg})$} & \multicolumn{2}{|c|}{ Age at surgery (years) } \\
\hline & $\overline{\text { Mean }(S D)}$ & Min-max & $\overline{\text { Mean }(S D)}$ & $\operatorname{Min}-\max$ \\
\hline Goniotomy & $28.8(4.7)$ & $24-46$ & $1.3(1.5)$ & $0.1-4.5$ \\
\hline Trabeculotomy & $27.7(3.1)$ & $20-32$ & $3.2(4.1)$ & $0.1-10.8$ \\
\hline Trabeculectomy MMC + trabeculotomy & $29.1(3.3)$ & $24-35$ & $0.7(0.7)$ & $0.0-2.2$ \\
\hline Trabeculectomy MMC & $25.5(2.3)$ & $22-30$ & $1.5(1.8)$ & $0.3-5.6$ \\
\hline
\end{tabular}

$I O P$ intra-ocular pressure, $S D$ standard deviation, Min-max minimum-maximum values

with mild severity (onset of glaucoma after 1 month of age) and with partially cloudy cornea were treated with goniotomy or trabeculotomy. Age and IOP at the time of surgery are reported in Table 3 . Mean age at the time of surgery, was 1.3 years (SD 1.5), 3.2 years (SD 4.1), 1.5 years (SD 1.8), and less than 1 year (mean 0.7, SD 0.7) for patients treated with goniotomy, trabeculotomy, trabeculectomy, and combined trabeculectomy and trabeculotomy, respectively.

Table 4 reports disease characteristics at baseline. IOP levels ranged from 22 to $26 \mathrm{mmHg}$ with a mean value of $23.6 \mathrm{mmHg}$ (SD 1.5). Vertical and horizontal corneal diameters ranged from 12.0 to $16.0 \mathrm{~mm}$ and from 11.5 to $16.0 \mathrm{~mm}$, respectively. Mean CCT was $561 \mu \mathrm{m}$ (SD 49; range 485-674 $\mu \mathrm{m}$ ). The preoperative and baseline mean CCT values were not significantly different (mean difference $3.3 \mu \mathrm{m}$; SD 16.0; Wilcoxon test $p$ value 0.186 )

At baseline, the IOP was measured in the sitting position in $11(31.4 \%)$ patients and in the supine position in $24(68.6 \%)$ patients (15 under general anesthesia; 9 awake).

\section{DISCUSSION}

GIPSy is a multicenter phase II interventional clinical trial that aims to evaluate the efficacy of
Table 4 Baseline ocular characteristics $(n=57)$

Baseline IOP $(\mathrm{mmHg})$

Mean (SD)

$23.6(1.5)$

Min-max

$22-26$

Vertical/horizontal corneal diameter (mm)

Mean (SD) $13.6(0.9) / 13.7$

Min-max

$12.0-16.0 /$

$11.5-16.0$

Central corneal thickness $(\mu \mathrm{m})$

Mean (SD)

$561(49)$

Min-max 485-674

Iris color, $n$ (\%)

Brown

$26(45.6)$

Blue/gray

$12(21.1)$

Dark brown

Green

$6(10.5)$

Green brown

$4(7.0)$

IOP intra-ocular pressure, $S D$ standard deviation, Min-max minimum-maximum values

medical treatment in a selected sample of predominantly Caucasian PPG patients partially responsive to a single surgical procedure. Patients with an IOP ranging from 22 to $26 \mathrm{~mm} \mathrm{Hg}$ after a single surgical procedure were included in the study. This is a frequent 
clinical condition in which the clinician tries to reach the target IOP by means of medical therapy. Higher levels of IOP are generally difficult to manage just with medical therapy, and are often treated with further surgery. Drugs chosen for the study are commonly used as first-line therapy for adult glaucoma [25], but until now systematic data have not been available on their use for the treatment of PPG. Thus, the choice of a prostaglandin analogue and carbonic anhydrase inhibitor was driven by the evidence of their efficacy in reducing IOP in adult glaucoma [26] and of their safety (local and systemic) [26, 27]. A goal of the current study was to define an effective therapeutic algorithm when a single surgical procedure was not able to achieve target IOP in patients affected by PPG.

The selection of the GIPSy population was defined in order to be highly representative of Caucasian PPG cases described in Europe. The mean age at presentation in the current study is comparable to that generally reported in the literature [28-31]. Published evidence on gender predilection of PPG are also in agreement with our data which show a female $\backslash$ male ratio equal to $1: 1.7$. In agreement with previously published literature, most of our patients $(80.0 \%)$ had bilateral glaucoma [13, 30, 32, 33].

Compared to healthy adults, children with glaucoma have been reported to have thinner corneas $[34,35]$. The mean CCT in the GIPSy was $561 \mu \mathrm{m}$ and ranged from 485 to $674 \mu \mathrm{m}$. To date, no systematic investigations on CCT have been performed in adequately sized samples of patients with pediatric glaucoma. In adults, the average corneal thickness is approximately $520 \mu \mathrm{m}$ at the center and approximately $700 \mu \mathrm{m}$ at the periphery [36]. The CCT in newborns was found to be greater than $580 \mu \mathrm{m}$ and decreased to adult values by 3 years of age $[37,38]$. Doughty et al. [39] found similar mean CCT values in children 5-15 years of age compared to adults older than 32 years of age. In a confocal microscopy study, Mastropasqua et al. [40] reported that the corneas in pediatric glaucoma patients have abnormal features in the Descemet membrane, stroma and endothelium. For example, the mean stromal keratocyte density was markedly reduced. The authors suggested that these findings were the direct result of corneal distention, which may explain why children with glaucoma have thinner corneas. Our data on CCT could be of interest in characterizing longitudinal changes in corneal thickness, and the eventual long-term effects of anti-glaucoma medications.

PPG patients recruited for this study were surgically treated according to current clinical practice. Angle surgeries (goniotomy and trabeculotomy) were performed in a large percentage of cases (61.4\%) [23].

The present study has limitations. First, the sample size is not particularly large. Recruiting adequately sized samples is an inevitable difficulty with trials investigating rare diseases, particularly when highly specialized centers and strict observation of study procedures are necessary. Second, the majority of participants were Caucasians. Consequently, the results of this study may not be generalizable to other ethnic groups.

By virtue of its design and population, the current study is representative of a clinical scenario encountered after the first surgery for PPG. Therefore, the results are expected to offer clinically relevant information on the utility of a defined pharmacological approach in the management of children with glaucoma.

\section{CONCLUSION}

The Glaucoma Italian Pediatric Study (GIPSy) investigated the use of a specific therapeutic 
algorithm with latanoprost and dorzolamide in pediatric glaucoma patients partially responsive to surgery. This article reports the study protocol and baseline characteristics of study participants.

\section{ACKNOWLEDGMENTS}

This study was supported by a grant from the Italian Medicines Agency (AIFA FARM7CZ54W 2007). All named authors meet the International Committee of Medical Journal Editors (ICMJE) criteria for authorship for this manuscript, take responsibility for the integrity of the work as a whole, and have given final approval to the version to be published. Authors commemorate their colleague Irene Floriani of the Mario Negri Institute, who passed away on 12 January 2016 and who dedicated her life to the research. She was involved in the statistical design and coordination of this clinical trial.

Disclosures. Elena Biagioli, Francesca Galli, Davide Poli, Eliana Rulli, Lital Hollander, Andreas Katsanos, Antonio Longo, Maurizio G. Uva and Valter Torri have nothing to disclose. Luciano Quaranta reports personal fees from Alcon, personal fees from Allergan, personal fees from Visufarma, personal fees from Bausch \& Lomb, outside the submitted work. Ivano Riva reports personal fees from Visufarma, personal fees from Angelini, outside the submitted work. Robert N. Weinreb declares the following: Consultant for Alcon, Allergan, Bausch\&Lomb, ForSight Vision5, Heidelberg Engineering, Optovue, Topcon; Research support from Genentech, Quark, National Eye Institute.

Compliance with Ethics Guidelines. The study was conducted in accordance with the
Declaration of Helsinki of 1964 as revised in 2013. The study protocol was approved by the Institutional Review Board of each centre. Parents provided written informed consent prior to any study-related activities.

Open Access. This article is distributed under the terms of the Creative Commons Attribution-NonCommercial 4.0 International License (http://creativecommons.org/licenses/ by-nc/4.0/), which permits any noncommercial use, distribution, and reproduction in any medium, provided you give appropriate credit to the original author(s) and the source, provide a link to the Creative Commons license, and indicate if changes were made.

\section{REFERENCES}

1. Alodhayb S, Morales J, Edward DP, Abu-Amero KK. Update on pediatric glaucoma. Semin Ophthalmol. 2013;28:131-43.

2. Beck A, Chang TCP. Section 1: definition and differential diagnosis. In: Weinreb RN, Grajewski A, Papadopulos M, Grigg J, Freedman S, editors. 9th World glaucoma association consensus meeting: childhood glaucoma. Amsterdam; 2013.

3. Papadopoulos M, Edmunds B, Fenerty C, Khaw PT. Childhood glaucoma surgery in the 21st century. Eye (Lond). 2014;28:931-43.

4. Luntz MH. The advantages of trabeculotomy over goniotomy. J Pediatr Ophthalmol Strabismus. 1984;21:150-3.

5. McPherson SD Jr, Berry DP. Goniotomy vs external trabeculotomy for developmental glaucoma. Am J Ophthalmol. 1983;95:427-31.

6. Allen L, Burian HM. Trabeculotomy ab externo. A new glaucoma operation: technique and results of experimental surgery. Am J Ophthalmol. 1962;53:19-26.

7. Elder MJ. Combined trabeculotomy-trabeculectomy compared with primary trabeculectomy for congenital glaucoma. $\mathrm{Br} \mathrm{J}$ Ophthalmol. 1994;78:745-8. 
8. Filous A, Brunova B. Results of the modified trabeculotomy in the treatment of primary congenital glaucoma. J AAPOS. 2002;6:182-6.

9. Mendicino ME, Lynch MG, Drack A, et al. Long-term surgical and visual outcomes in primary congenital glaucoma: 360 degrees trabeculotomy versus goniotomy. J AAPOS. 2000;4:205-10.

10. Mullaney PB, Selleck C, Al-Awad A, Al-Mesfer S, Zwaan J. Combined trabeculotomy and trabeculectomy as an initial procedure in uncomplicated congenital glaucoma. Arch Ophthalmol. 1999;117:457-60.

11. O'Connor G. Combined trabeculotomy-trabeculectomy for congenital glaucoma. Br J Ophthalmol. 1994;78:735.

12. Tanimoto SA, Brandt JD. Options in pediatric glaucoma after angle surgery has failed. Curr Opin Ophthalmol. 2006;17:132-7.

13. Papadopoulos M, Cable N, Rahi J, Khaw PT, Investigators BIGES. The British infantile and childhood glaucoma (BIG) eye study. Invest Ophthalmol Vis Sci. 2007;48:4100-6.

14. Ott EZ, Mills MD, Arango S, et al. A randomized trial assessing dorzolamide in patients with glaucoma who are younger than 6 years. Arch Ophthalmol. 2005;123:1177-86.

15. Donohue EK, Wilensky JT. Trusopt, a topical carbonic anhydrase inhibitor. J Glaucoma. 1996;5:68-74.

16. Portellos M, Buckley EG, Freedman SF. Topical versus oral carbonic anhydrase inhibitor therapy for pediatric glaucoma. J AAPOS. 1998;2:43-7.

17. Raber S, Courtney R, Maeda-Chubachi T, et al. Latanoprost systemic exposure in pediatric and adult patients with glaucoma: a phase 1, open-label study. Ophthalmology. 2011;118:2022-7.

18. Maeda-Chubachi T, Chi-Burris K, Simons BD, et al. Comparison of latanoprost and timolol in pediatric glaucoma: a phase 3, 12-week, randomized, double-masked multicenter study. Ophthalmology. 2011;118:2014-21.

19. Black AC, Jones S, Yanovitch TL, et al. Latanoprost in pediatric glaucoma-pediatric exposure over a decade. J AAPOS. 2009;13:558-62.

20. Chang L, Ong EL, Bunce C, et al. A review of the medical treatment of pediatric glaucomas at Moorfields Eye Hospital. J Glaucoma. 2013;22:601-7.
21. Uva MG, Avitabile T, Reibaldi M, et al. Long-term efficacy of latanoprost in primary congenital glaucoma. Eye (Lond). 2014;28:53-7.

22. Ravinet E, Mermoud A, Brignoli R. Four years later: a clinical update on latanoprost. Eur J Ophthalmol. 2003;13:162-75.

23. Papadopoulos M, Edmunds B, Chiang M, Mandal A, Grajewsky AL, Khaw PT. Section 5: glaucoma surgery in children. In: Weinreb RN, Grajewski A, Papadopulos M, Grigg J, Freedman S, editors. 9th World glaucoma association consensus meeting: childhood glaucoma. 2013.

24. Blumberg $\mathrm{D}$, Congdon $\mathrm{N}$, Jampel $\mathrm{H}$, et al. The effects of sevoflurane and ketamine on intraocular pressure in children during examination under anesthesia. Am J Ophthalmol. 2007;143:494-9.

25. European Glaucoma Society. Terminology and guidelines for glaucoma. IV ed. Savona: PubliComm; 2014.

26. Schmidl D, Schmetterer L, Garhofer G, Popa-Cherecheanu A. Pharmacotherapy of glaucoma. J Ocul Pharmacol Ther. 2015;31:63-77.

27. Kanner E, Tsai JC. Glaucoma medications: use and safety in the elderly population. Drugs Aging. 2006;23:321-32.

28. Gencik A, Gencikova A, Ferak V. Population genetical aspects of primary congenital glaucoma. I. Incidence, prevalence, gene frequency, and age of onset. Hum Genet. 1982;61:193-7.

29. Aponte EP, Diehl N, Mohney BG. Incidence and clinical characteristics of childhood glaucoma: a population-based study. Arch Ophthalmol. 2010;128:478-82.

30. MacKinnon JR, Giubilato A, Elder JE, Craig JE, Mackey DA. Primary infantile glaucoma in an Australian population. Clin Exp Ophthalmol. 2004;32:14-8.

31. Taylor RH, Ainsworth JR, Evans AR, Levin AV. The epidemiology of pediatric glaucoma: the Toronto experience. J AAPOS. 1999;3:308-15.

32. Mandal AK, Chakrabarti D. Update on congenital glaucoma. Indian J Ophthalmol. 2011;59(Suppl):S148-57.

33. Alanazi FF, Song JC, Mousa A, et al. Primary and secondary congenital glaucoma: baseline features from a registry at King Khaled Eye Specialist Hospital, Riyadh, Saudi Arabia. Am J Ophthalmol. 2013;155:882-9. 
34. Wygnanski-Jaffe T, Barequet IS. Central corneal thickness in congenital glaucoma. Cornea. 2006;25:923-5.

35. Henriques MJ, Vessani RM, Reis FA, et al. Corneal thickness in congenital glaucoma. J Glaucoma. 2004;13:185-8.

36. Doughty MJ, Zaman ML. Human corneal thickness and its impact on intraocular pressure measures: a review and meta-analysis approach. Surv Ophthalmol. 2000;44:367-408.

37. Ehlers N, Sorensen T, Bramsen T, Poulsen EH. Central corneal thickness in newborns and children. Acta Ophthalmol (Copenh). 1976;54:285-90.
38. Remon L, Cristobal JA, Castillo J, et al. Central and peripheral corneal thickness in full-term newborns by ultrasonic pachymetry. Invest Ophthalmol Vis Sci. 1992;33:3080-3.

39. Doughty MJ, Laiquzzaman M, Muller A, Oblak E, Button NF. Central corneal thickness in European (white) individuals, especially children and the elderly, and assessment of its possible importance in clinical measures of intra-ocular pressure. Ophthalmic Physiol Opt. 2002;22:491-504.

40. Mastropasqua L, Carpineto $\mathrm{P}$, Ciancaglini $\mathrm{M}$, Nubile M, Doronzo E. In vivo confocal microscopy in primary congenital glaucoma with megalocornea. J Glaucoma. 2002;11:83-9. 